\title{
Les travaux du groupe Association H. Capitant des Amis de la Culture Juridique Française/Société de législation comparée: terminologie, principes directeurs et révision des Principes du droit européen du contrat
}

\author{
Bénédicte Fauvarque-Cosson
}

Published online: 22 August 2008

(C) ERA 2008

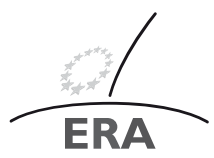

EUROPÄISCHE RECHTSAKADEMIE ACADEMY OF EUROPEAN LAW ACADEMIE DE DROIT EUROPEEN ACAOEME DE OROT EUROPEEN ACCADEMIA DI DIRITTO EUROP
TRIER - TREVES - TREVIRI

\section{Présentation du groupe}

Le Groupe commun Association Henri Capitant des Amis de la Culture Juridique Française/Société de législation comparée (ci-après dénommé AHC/SLC) a été coordonné par les secrétaires généraux de ces deux associations, à savoir Denis Mazeaud et moi-même. Ce groupe a travaillé sur les trois tiroirs de cette «boîte à outils» que devrait être le cadre commun de référence (CCR): les principes directeurs du droit des contrats, la terminologie et des règles modèles. Une version modifiée des Principes du droit européen du contrat, élaborés par la Commission Lando ${ }^{1}$, est ainsi proposée, dans un esprit de modernisation mais aussi et surtout de fidélité à la structure de l'œuvre, devenue un modèle de référence en Europe et au-delà. L'intégralité de ces travaux est publiée par ces associations, dans la collection «droit privé comparé et européen» de la Société de législation comparée, en deux volumes. L'un contient la terminologie contractuelle commune ${ }^{2}$; l'autre, intitulé «Principes contractuels communs» rassemble les Principes directeurs du droit européen du contrat ainsi que le projet de révision des «Principes du droit européen du contrat» ${ }^{3}$. Une grande partie des travaux a été traduite en anglais (l'intégralité sauf les notes et commentaires

\footnotetext{
1) Société de législation comparée [6].

2) Association H. Capitant et Société de législation comparée [2].

3) Association H. Capitant et Société de législation comparée [3]. En juillet 2008, ces ouvrages, traduits en anglais, seront publiés par l'éditeur Sellier: Association H. Capitant et Société de législation comparée [1].
}

Bénédicte Fauvarque-Cosson $(\bowtie)$

Professeur à l'Université Panthéon-Assas (Paris II),

Secrétaire Générale de la Société de législation comparée

97 rue des Tennerolles, 92210 Saint-Cloud, France

e-mail: b.fauvarquecosson@wanadoo.fr

Cet article a été présenté lors d'un séminaire de 1'ERA portant sur «Le Projet de Cadre Commun de Référence», organisé en coopération avec le Joint Network on European Private Law les 6 et 7 mars 2008 à Trèves. 
comparatifs qui accompagnent les propositions de règles modèles nouvelles) et sont désormais publiés par l'éditeur sellier European Law Publishers dans un ouvrage intitulé: European Contract Law: Materials for the CFR, Terminology, Guiding Principles, Evaluation of PECL.

D'un point de vue politique, le projet de révision des Principes du droit européen du contrat sera sans doute, parmi les trois volets des travaux réalisés par le groupe $\mathrm{AHC} / \mathrm{SLC}$, celui qui retiendra le plus l'attention. Cependant, conformément à la mission impartie par la Commission (élaborer une boîte à outils, composée de trois tiroirs, l'un portant sur les principes, l'autre sur les définitions et la terminologie, le troisième sur des règles modèles), notre groupe a aussi travaillé sur ces deux premiers volets. Aujourd'hui, ce travail apparaît d'autant plus utile qu'il complète celui fait par les rédacteurs du DCFR qui ont essentiellement fait porter leurs efforts sur l'élaboration de règles modèles.

Sur un plan terminologique, le DCFR ne contient pas, pour le moment, d'étude de fond des termes et concepts utilisés. En revanche, il comprend une Annexe fort précieuse de définitions; mais cette annexe n'est véritablement utile qu'en lien avec l'utilisation des règles modèles énoncées par le DCFR. Le travail du groupe AHC/SLC, plus précisément l'ouvrage, de plus de 500 pages, intitulé «Terminologie contractuelle commune», est donc très complémentaire. Il servira, d'une part, à l'élaboration finale du CCR et, d'autre part, à l'utilisation et la traduction du DCFR par tous les juristes des 27 Etats membres.

Les principes directeurs proposés par le groupe AHC/SLC en tête de l'ouvrage intitulé «Principes contractuels communs» concernent le seul droit des contrats. Ils ont vocation à guider l'interprétation et l'application des Principes du droit européen du contrat auxquels ils sont associés. Aussi prennent-ils la forme d'un chapitre préliminaire, construit sur les trois piliers suivants: la liberté, la sécurité et la loyauté contractuelles, piliers qui se retrouvent dans tous les droits des Etats de l'Union européenne, en des formes variables. Afin de donner un contenu concret à ces concepts, l'étude a été menée dans une perspective comparative, portant sur un grand nombre de droits internes, sur le droit international et communautaire ainsi que sur d'autres codifications doctrinales: Principes Unidroit, avant-projet de code européen des contrats, avant-projet de réforme du droit français des obligations et de la prescription.

C'est cette même perspective comparative qui a constamment guidé les travaux du troisième sous-groupe, celui chargé de la révision des PECL.

\section{Le choix du groupe AHC/SLC: la révision de PECL}

\subsection{Pourquoi ce choix}

A l'origine, le groupe AHC/SLC avait pour mission de réfléchir aux principes fondamentaux qui irriguent le droit européen des contrats, et de travailler sur la terminologie contractuelle commune, ceci dans la perspective d'aider à la préparation des deux premiers «tiroirs» du cadre commun de référence. Puis, lors d'une rencontre à Paris, entre le professeur Christian von Bar et les représentants de nos deux Associations, ce dernier nous a suggéré de concentrer nos efforts sur les règles modèles, de proposer de nouveaux textes. Comme il était difficile de travailler sur le DCFR, encore 
en cours d'élaboration, d'un commun accord, nous avons pris pour base de travail le modèle des PDEC.

Chacun ici en conviendra: les Principes du droit européen du contrat issus de la Commission présidée par le Professeur Ole Lando constituent une œuvre doctrinale majeure. Aux termes de leur article 1:101 (1) intitulé «Application des principes»: Les présents Principes sont destinés à s'appliquer en tant que règles générales $d u$ droit des contrats dans l'Union européenne». Et de fait, ces Principes servent de modèle législatif, en Europe et dans le monde entier. Les juges nationaux s'y réfèrent, les juges européens également. La référence aux PDEC est parfois simplement persuasive; d'autres fois, elle acquiert une plus forte connotation normative. Les conclusions rendues par l'avocat général ${ }^{4}$ sur un arrêt de la CJCE du 28 juin $2007^{5}$, dans le domaine de l'interprétation d'un contrat, sont à cet égard très significatives: «Il faut en droit communautaire, pour l'interprétation des déclarations de volonté, recourir aux principes généraux de droit. Les dispositions sur les principes généraux de droit relatifs à l'interprétation des déclarations de volonté peuvent être tirées du chapitre 5 intitulé "Interprétation» de l'ouvrage "Principles of European Contract Law» (Principes du droit européen des contrats). Il s'agit d'un ouvrage qui couvre le fond commun du droit contractuel des États membres».

Il était donc non seulement souhaitable mais absolument naturel de prendre les PDEC comme point de départ des travaux relatifs au cadre commun de référence. C'est du reste ce qui a également été fait par les groupes qui ont élaboré le DCFR. Simplement, le groupe AHC/SLC est resté beaucoup plus fidèle à la structure même de l'œuvre accomplie. Cela s'explique par le fait que nous avons travaillé sur le seul droit des contrats et non pas sur l'ensemble du droit privé européen.

\subsection{Comment le groupe AHC/SLC a-t-il procédé?}

Trois sous-groupes ont successivement été constitués, afin d'effectuer des recherches dans les trois directions voulues par la Commission (les trois «tiroirs» du CCR): un groupe chargé de travailler sur la terminologie et un groupe chargé de proposer des principes directeurs du droit des contrats en Europe ont été créés. Un troisième groupe, constitué un peu plus tard pour les raisons ci-dessus exposées, a reçu mission de réviser les Principes du droit européen du contrat ${ }^{6}$.

L'analyse de chaque article des PDEC est enrichie d'une discussion, elle-même composée d'un commentaire de l'article puis d'une étude comparée, avec notamment, comme points de comparaison, les Principes Unidroit relatifs aux contrats du commerce international, la Convention de Vienne de 1980 sur la vente internationale de marchandises, l'avant-projet de code européen des contrats (dit Gandolfi), l'avantprojet de réforme du droit des obligations et de la prescription, le Draft Common Frame of Reference. Viennent ensuite les propositions de textes nouveaux, précédées d'une motivation.

\footnotetext{
4) Mme Verica Trstenjak, conclusions du 6 mars 2007.

5) Arrêts du 28 juin 2007, Bonn Fleisch (C-1/06, Rec. p. I-5609).

6) L'ensemble des travaux a été coordonné par Denis Mazeaud et moi-même. Les trois groupes, respectivement dirigés par Aline Tenenbaum, Guillaume Wicker et Jean-Baptiste Racine, ont travaillé avec des équipes de jeunes professeurs et enseignants-chercheurs. Les noms des participants figurent sur chacun des ouvrages.
} 
Le groupe de travail a ainsi mené à bien, avec tout le respect qui s'impose, la révision des principes du droit européen du contrat, «œuvre monumentale, historique, majeure» ${ }^{7}$. Si, sur de nombreux points, des aménagements sont apparus souhaitables, dans le fond comme dans la forme, un bouleversement d'ensemble n'a pas paru s'imposer. C'est ici que se situe la plus grande divergence entre l'optique retenue par le DCFR et celle du groupe français.

\section{Les différences d'approche par rapport au DCFR}

Certains choix ont été faits; ils diffèrent de ceux retenus pour élaborer le DCFR.

3.1 Le travail de révision se limite au seul droit des contrats.

Tous les contrats sont visés qu'ils soient conclus entre professionnels ou entre un professionnel et un consommateur ou entre consommateurs. Les contrats spéciaux ne sont pas inclus.

De ce fait, nous n'avons pas rencontré les mêmes difficultés terminologiques, ni les mêmes difficultés relatives au plan, que celles affrontées par les rédacteurs du DCFR.

\subsection{Le plan est simple, universel.}

Nous avons adopté un plan simple, connu, au demeurant très proche de celui diffusé par la Commission dans l'annexe à son Plan d'action. Ce plan suit celui retenu par les PDEC, avec quelques modifications.

3.3 Le Chapitre premier de ce projet de révision des PDEC contiendra les principes directeurs du droit des contrats.

En l'absence de principes directeurs commentés et expliqués, tout et son contraire pourra toujours être dit sur l'esprit du droit européen des contrats, qu'il s'agisse de l'acquis communautaire ou des règles modèles contenues dans le futur cadre commun de référence. Un manifeste publié par un groupe d'universitaires dénommé «justice sociale», largement diffusé, a mis en exergue l'importance de la question ${ }^{8}$. Parce qu'il concerne les relations sociales et économiques entre citoyens, l'enjeu est éminemment politique. L'édification d'un socle juridique commun en Europe passe donc d'abord par l'élaboration de Principes directeurs du droit européen du contrat. C'est à la lumière de ces principes que les règles modèles devraient être élaborées, et non l'inverse.

\footnotetext{
7) Introduction de J.-B. Racine et G. Wicker, dans Association H. Capitant et Société de législation comparée [3].

8) Ce Manifeste propose un «programme de justice sociale» pour un droit européen des contrats et critique au passage le processus retenu par la Commission européenne pour l'élaboration du cadre commun de référence, jugé insuffisamment démocratique: Study Group on Social Justice in European Private Law [7]; Groupe d'étude sur la justice sociale en droit privé européen [4].
} 
Certes, l'introduction au DCFR donne quelques informations sur les objectifs et

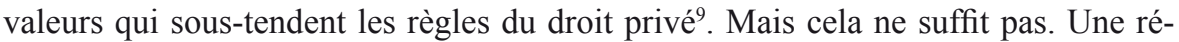
flexion plus aboutie, sur les fondements philosophiques du droit des contrats, est apparue nécessaire. C'est ce que nous avons entrepris, en la forme de principes directeurs, assortis de commentaires et notes comparatives.

Cette réflexion trouve naturellement sa place dans un projet qui part des PDEC pour les moderniser, à la lumière du droit comparé. Les textes qui ont été proposés pourraient également être introduits dans le DCFR car, sur le fond, ils sont dans l'ensemble compatibles avec l'esprit du DCFR (le plus souvent, c'est le cas). La difficulté vient néanmoins du fait que ces principes directeurs ne concernent que le seul droit des contrats, tandis que le DCFR a une vocation beaucoup plus large. Sous cette réserve, leur insertion au sein du DCFR, pourrait se faire, soit en la forme de considérants, soit en celle de textes directeurs. Le groupe AHC/SLC exprime ici sa préférence pour la deuxième solution. Cela permettrait de donner une armature au Livre I du DCFR, actuellement assez décevant. Rappelons à cet égard l'appréciation critique d'Ole Lando: on aurait pu espérer un début «more appealing», avec, notamment, des principes fondamentaux, selon le modèle des PDEC et des Principes Unidroit ${ }^{10}$.

\subsection{Les textes sont concis}

Souvent, les textes des PECL ont été révisés à des fins de clarté et de concision, sans altération de leur contenu. Les textes proposés évitent de rentrer dans trop de détails. L'objectif a toujours été de présenter des textes clairs, aisément accessibles pour le citoyen et non uniquement compréhensibles par des experts du droit européen des contrats.

Ainsi, la question n'est pas tant de savoir quel modèle a trouvé le meilleur équilibre mais plutôt quel modèle nous voulons en Europe: simple, flexible, adaptable aux cas d'espèce ou complexe, plus rigide et très détaillé. Le législateur doit-il tout prévoir? Je ne critique pas les textes du DCFR: je pose simplement la question: quel modèle, en Europe, pour le droit européen des contrats? Trop de détails, trop de textes tuent l'accès au droit.

\subsection{L'étendue de la révision}

La table des matières de l'ouvrage permet de saisir, d'un coup d'œil rapide, les principales modifications, les textes actuels et ceux proposés étant systématiquement mis en perspective. Tous les chapitres des PDEC n'ont pas été analysés, soit parce que le temps pressait, soit parce que certains (notamment la prescription) ne sont pas spécifiques au droit des contrats. Ainsi, le Chapitre I (dispositions générales), le Chapitre 10 (pluralité de sujets), le Chapitre 11 (cession de créance), 13 (compensation), 14 (prescription), 17 (capitalisation des intérêts) ont été laissés de côté. Il nous a semblé nécessaire, en effet, de nous concentrer sur le noyau dur: formation du contrat, validité, interprétation, contenu, effets, exécution et inexécution, changement de parties.

\footnotetext{
9) Voir infra 5.2

10) Lando [5], p. 249.
} 


\subsection{La nouvelle structure}

Les Chapitres 2, 3 et 5 des PDEC conservent leurs intitulés. Le Chapitre 4 (validité) et le Chapitre 15 (illicéité) sont fondus dans un nouveau chapitre désormais intitulé «Invalidité».

Le Chapitre 6 des PDEC «Contenu et effets» est divisé en deux si bien que le Chapitre 7 sur «l'exécution» devient le Chapitre 8; il accueille le Chapitre 16 relatifs aux «conditions». Les Chapitres 8 et 9 deviennent les Chapitres 9 et 10 . Un nouveau Chapitre 11, intitulé «Changement de parties», accueille les textes des PDEC sur le sujet (soit la «cession de créance», Chapitre 11 PDEC et la «substitution d'un nouveau débiteur et cession de contrat» Chapitre 12 PDEC).

La synthèse des nouveaux textes proposés contient près de 200 articles.

Il reste à présenter, point par point, les grands changements proposés par rapport au modèle des PDEC.

\section{Comparaison des nouvelles règles et des PDEC}

Cette présentation, non exhaustive, explore certains des principaux changements.

\subsection{La formation du contrat}

Le Chapitre 2 sur la formation du contrat a fait l'objet de remaniements visant à mieux mettre en lumière la phase précontractuelle, devenue essentielle dans la pratique des affaires.

Les règles relatives à la négociation et aux pourparlers sont ainsi placées en tête du Chapitre, alors que dans les PDEC, elles sont traitées à la fin de ce Chapitre, sous le seul angle de la responsabilité en cas de rupture des négociations. D’emblée, il est précisé que les négociations précontractuelles sont soumises aux devoirs suivants: bonne foi, information, confidentialité. Le devoir de confidentialité est repris, tel qu'il figurait dans les PDEC. Le devoir de bonne foi fait l'objet d'un texte qui modifie l'article 2:301 relatif aux négociations contraires à la bonne foi. Le devoir d'information fait l'objet d'un article 2:102 nouveau. L'alinéa 3 de cet article renforce simplement le devoir d'information de «la partie qui dispose d'une compétence technique particulière».

En ce qui concerne la conclusion du contrat, l'article 2:102 intitulé «Intention» est modifié afin de mieux éclairer le principe selon lequel un contrat n'est conclu que si les parties manifestent l'intention d'être liées juridiquement. Un nouvel alinéa premier ${ }^{11}$ ajoute qu'il faut avoir «manifesté son intention d'être engagé» pour l'être effectivement. Dans les PDEC, le texte suivant traitait de 1' "Accord suffisant»; il est remplacé par un texte intitulé «Accord sur les éléments essentiels du contrat» ${ }^{12}$. L'article précise comment déterminer ces «éléments essentiels». Un certains nombre de textes suivants font l'objet d'aménagements et de réécritures plus ou moins formelles, sans remise en cause des grandes options de fond.

\footnotetext{
11) Article $2: 202:$ Intention (article $2: 102$ modifié)

${ }^{12)}$ Article $2: 203$ ajout se substituant à l'article $2: 103$ : Accord suffisant
} 


\subsection{La représentation}

En ce qui concerne la représentation, un choix de fond a été fait: le chapitre révisé régit non seulement les rapports externes (représentant-tiers) mais aussi internes (représenté-représentant). Cela est précisé dans le nouvel article 3:101. Toutefois, dans la mesure où ce Chapitre ne porte que sur le «pouvoir de représentation» et non sur la représentation en général, il va de soi que toutes les relations entre représentant et représenté n'ont pas vocation à être couvertes (ainsi restent par exemple exclues les questions de reddition des comptes). La plupart des textes ont fait l'objet de modifications: d'autres ont été ajoutés, tel celui sur l'insolvabilité de l'intermédiaire ou l'inexécution essentielle à l'égard du représenté et à l'égard du tiers (art. 3:301 et 302).

Le DCFR a modifié les PDEC sur la représentation, sans toutefois étendre son domaine d'application aux relations externes. Sur le plan terminologique, il propose, par souci de cohérence, d'utiliser le mot représentant («representative») en lieu et place de «représentant» et «intermédiaire»

\subsection{L'invalidité}

Le Chapitre 4, intitulé Invalidité, reprend les Chapitres 4 et 15 des PDEC qui traitent, l'un et l'autre, des causes d'invalidité du contrat (erreur, dol, violence, lésion qualifiée) et le second de l'illicéité. Une section I pose des dispositions générales, une section II traite de l'invalidité pour vices du consentement, une section 3 de l'invalidité pour illicéité (elle est divisée en trois paragraphes: atteinte aux principes fondamentaux, atteinte à une règle impérative, atteinte aux droits des tiers). Dans une section 4, un régime commun est proposé pour la mise en œuvre de l'invalidité et de ses conséquences (avec toutefois des dispositions spéciales en cas d'atteinte aux principes fondamentaux).

Ce remaniement rejoint celui opéré par le DCFR (Livre II, chap. 7, causes d'invalidité) mais il va plus loin avec l'élaboration de dispositions communes quant aux conséquences de l'invalidité, ce qui a permis d'éviter certaines répétitions.

Les textes sur l'erreur ont été remaniés afin d'apporter plus de simplification. Un texte introductif (l'article 4:201, alinéa 1) a été ajouté, qui fait référence à la nullité «lorsque le consentement d'une partie a été vicié». De plus, ce texte intègre l'article 4:116 des PDEC, qu'il réécrit en partie, relatif à la nullité partielle, dont les conditions de mise en œuvre et le régime sont ainsi précisés.

Le texte sur l'adaptation du contrat est réécrit; celui sur l'information inexacte est réintégré aux Chapitre 2 (art. 2:102). Le texte sur le dol est également révisé; celui sur la contrainte est modifié.

Quant à l'article sur le «profit excessif ou avantage déloyal» (4:109 PDEC), qui introduit la lésion qualifiée, il est réintitulé «Avantage manifestement excessif» (art. 4:207). Le mot «avantage», plus large, permet mieux de viser certains déséquilibres non pécuniaires et l'adverbe «manifestement» vise à cantonner cette cause de nullité. Le style, plus direct, est allégé.

L'article 4:110 des PDEC, relatif aux «Clauses abusives qui n'ont pas été l'objet d'une négociation individuelle» fait également l'objet de certaines révisions et restrictions; une nouvelle rédaction est proposée, qui donne à la victime du déséquilibre la faculté de saisir le juge afin qu'il révise la clause au lieu de l'annuler (comp. DCFR 
II.-9:103, qui prévoit simplement que ces clauses ne peuvent pas toujours être opposées par leur bénéficiaire)

Le principe de l'annulation par voie de notification (que tous les systèmes juridiques ne connaissent pas) est maintenu mais les textes proposés apportent des précisions ou améliorations, notamment en ce qui concerne le régime de l'annulation, la forme, les délais et le cas où, en l'absence de notification, l'annulation est soulevée devant le juge, comme un moyen de défense. Le principe est par ailleurs étendu à l'inefficacité du contrat résultant de son illicéité, ce qui explique le choix du terme «invalidation par notification» au lieu d'annulation. En cas d'atteinte aux droits fondamentaux, la notification n'est pas nécessaire. Par ailleurs, un texte nouveau prévoit la possibilité d'ouvrir à l'autre partie une action interrogatoire afin qu'elle puisse dissiper l'incertitude sur l'éventuelle invalidation par notification du contrat. Après que l'autre partie lui a demandé de dire s'il exercerait ou non son droit, et passé un certain délai, le titulaire du droit d'invalider le contrat sera donc déchu de ce droit (art. 4:406 ajouté).

En ce qui concerne les effets de l'invalidité, six nouveaux textes ont été élaborés afin de poser un régime applicable aux restitutions (comp. l'article 7:212 DCFR qui recherche une cohérence avec les nouvelles règles relatives à l'enrichissement injuste et au transfert de propriété des biens meubles, v. Principes p. 397).

Enfin, la section 3 sur l'invalidité pour illicéité a également fait l'objet d'ajouts et de révisions des textes existants.

\subsection{L'interprétation}

Les règles générales sur l'interprétation, telles qu'elles figurent dans les PDEC, constituent un bon compromis entre l'approche subjective du droit français et celle, objective, des pays de tradition de common law. Sur un point précis, les textes révisés proposent une modification importante, inspirée des tendances actuelles et, notamment, de l'Avant-projet français de réforme du droit des obligations et de la prescription: il s'agit de l'interprétation en faveur de la partie faible. Ainsi, l'article 5:105, intitulé «interprétation préférentielle», modifie l'article 5:103 PDEC par extension du champ d'application de la règle contra proferentem à d'autres cas que ceux où les clauses du contrat n'ont pas été l'objet d'une négociation: «Lorsque la loi contractuelle a été établie sous l'influence dominante d'une partie, on doit, dans le doute, l'interpréter en faveur de l'autre. En particulier, les clauses du contrat s'interprètent de préférence contre celui qui les a proposées».

\subsection{Le contenu}

Le Chapitre 6 des PDEC ne traite plus que du contenu, tandis que les effets sont examinés dans le chapitre suivant. Un nouvel article, intitulé «détermination du contenu» ouvre ce Chapitre 6. Il précise ce que l'on entend par «contenu»: «Le contenu du contrat est composé d'obligations expresses ou implicites. Les éléments du contenu sont notamment la qualité de la prestation, les délais d'exécution et le prix».

Des textes relatifs à la durée sont introduits (dans les PDEC, la question est essentiellement traitée sous l'angle de la résiliation, art. 6:109 PDEC). Il est suggéré d'introduire une «durée minimale raisonnable eu égard à l'économie générale $d u$ contrat», nécessaire à la viabilité économique du contrat au regard de l'importance des 
investissements et du temps nécessaire pour les amortissements (art. 6:105 nouveau, Principes, p. 492). Un article nouveau traite également de la chose future (6:110).

L'article 6:101 des PDEC «Déclarations donnant naissance à des obligations» est déplacé et révisé car il était fort long et complexe. Le nouvel article 6:102 traite des obligations implicites et contient, dans un alinéa 2, la réécriture de 6:101. Surtout, à sa suite, un article 6:103 vient consacrer la distinction entre obligations de moyens et de résultat qui ne figurait jusqu'alors que dans les commentaires des PDEC (mais avait déjà été consacrée par les Principes d'Unidroit).

Les textes sur la détermination du prix sont à peine retouchés.

\subsection{Les effets}

Le Chapitre 7 relatif aux Effets comprend le changement de circonstances, des textes sur la durée, la simulation, la stipulation pour autrui.

Dans le chapitre sur le contenu, la durée est appréhendée sous l'angle de l'économie générale du contrat; dans celui sur les effets, elle l'est sous l'angle de la rupture et de la prolongation du contrat (principes, p. 513). Les articles 7:103 à 7:106 sur la durée sont particulièrement innovants.

En ce qui concerne le changement de circonstances, les grandes orientations demeurent les mêmes, avec le pouvoir du juge d'adapter le contrat. La rédaction est néanmoins beaucoup plus concise ${ }^{13}$; un nouveau texte (article 7:102) est ajouté. Il concerne les clauses relatives à la répartition des risques.

\subsection{L'exécution}

Le Chapitre 8, intitulé «Exécution», intègre une nouvelle section (Section 2) avec des textes sur la condition et d'autres sur les «délais d'exigibilité» (le mot «terme» n'a pas été retenu car il est difficile à traduire en anglais et n'a pas la même signification). Les textes sur la condition sont enrichis de nouveaux textes, notamment sur la source de la condition, le rapport des parties pendente conditione, les effets de la réalisation de la condition suspensive et résolutoire (le DCFR n'a pas procédé à des changements majeurs).

\subsection{L'inexécution et les divers moyens en cas d'inexécution (Chapitres 9 et 10)}

Dans le Chapitre 10, on signalera l'encadrement, plus étroit, de la faculté de résoudre par anticipation le contrat (art. 10:308, p. 709) et l'ajout d'un texte sur les clauses résolutoires (10:309). Ce texte précise les conditions de leur efficacité et prévoit notamment qu'elles «doivent expressément désigner les engagements dont l'inexécution entraînera la résolution du contrat». Le texte relatif aux effets de la résolution (art. 9:305) est également revu et le principe est modifié: le principe de non-rétroactivité est écarté, sans pour autant que soit posé celui de la rétroactivité. La règle de principe est la suivante (art. 10:310, p. 713): «Dans les conditions prévues par la présente section, la résolution du contrat oblige chacune des parties à restituer ce qu'elle a reçu et, le cas échéant, à indemniser son cocontractant».

\footnotetext{
${ }^{13)}$ Article $7: 101$ : Changement de circonstances
} 


\subsection{Les changement de parties}

Le Chapitre 11 intitulé «changement de parties» opère des regroupements opportuns. Un seul chapitre, avec trois sections relatives à la cession de créance, à la substitution de débiteur et à la cession de contrat est créé. Une approche semblable a été retenue par le DCFR.

\section{Comparaison des textes révisés et du DCFR}

\subsection{La structure}

L'introduction du DCFR expose les raisons de l'approche large retenue, consistant à proposer un modèle qui va bien au-delà du seul droit des contrats et dont le pilier central est le droit des obligations: dans la mesure où le droit des contrats fait partie du droit privé, il faut pouvoir tester les règles retenues sur les principaux contrats spéciaux et, le cas échéant, les ajuster en conséquence ${ }^{14}$. Si le DCFR aborde le droit des obligations comme une entité («organic entity or unit»), c'est en raison du fait, expliquent les auteurs de l'introduction, que les frontières entre le droit des contrats et les autres branches du droit sont souvent difficiles à tracer.

Le livre I du DCFR s'intitule «General provisions». Il est encore peu fourni et se compose pour le moment de 6 articles uniquement. Il pourrait intégrer certains des principes directeurs du droit des contrats. Cela serait même assez opportun. Il aurait en effet été parfaitement conforme à la mission conférée par la Commission européenne d'intégrer de tels principes: d'après le Plan d'Action, c'est le premier tiroir du CCR. Par contraste, le Chapitre I des Principes du droit européen du contrat, intitulé «General provisions» est composé de trois sections (l'une sur l'objet des principes, l'autre sur les devoirs généraux incluant devoirs de bonne foi et de collaboration, la troisième sur la terminologie et autres dispositions).

Les Livres II et III du DCFR, respectivement intitulés «Contracts and other juridical acts» et «Obligations and corresponding rights», reprennent en grande partie les textes des PDEC. Le Livre IV, intitulé «Specific contracts and the rights and obligations arising from them» traite de la vente, du bail de biens meubles, du contrat de services, du mandat, des contrats d'agence commerciale, de franchise et de distribution, des prêts (en préparation), des sûretés personnelles. Le DCFR pourrait ultérieurement intégrer les textes élaborés en droit des assurances mais il n'est pas certain que les travaux menés par le groupe droit des assurances puissent être intégrés en l'état.

Le Livre II du DCFR traite des contrats et autres actes juridiques (ce livre reprend les premiers chapitres des PDEC); le Livre III régit les «obligations et droits correspondants»: il s'applique aussi bien aux obligations d'origine contractuelle qu'aux autres. Les arguments en faveur du système nouveau sont parfaitement exposés par les auteurs de l'introduction ${ }^{15}$ : le contrat est un accord, un type d'acte juridique parmi d'autres, également régis par le Livre II. Il est dissocié de la «relation juridique»,

\footnotetext{
${ }^{14)}$ von Bar/Clive/Schulte-Nölke [8], Introduction, p. 20, §42: "Contract law as part of private law".

${ }^{15)}$ von Bar/Clive/Schulte-Nölke [8], Introduction, p. 22.
} 
construite à partir d'obligations et de droits respectifs. Or cette relation juridique ne résulte pas nécessairement du contrat si bien que le Livre III a également vocation à régir les obligations non contractuelles. Un certain nombre des articles du Livre III concernent néanmoins le seul droit des contrats.

Le plan des PDEC a ainsi été démantelé. L'exhaustivité l'a emporté sur la simplicité. C'est un choix. Indiscutablement, les Livres I, II, III chahutent le modèle contractuel européen et international. Or les PDEC et les Principes Unidroit constituent deux modèles de plus en plus connus et utilisés (notamment par les législateurs mais aussi par les juges et les arbitres) dans le monde entier. Ils adoptent l'un et l'autre un plan simple, chronologique, familier à beaucoup de droits nationaux. Fallait-il bouleverser cette structure au profit d'une autre, déroutante pour beaucoup? D'après Ole Lando, la méthode abstraite, d'inspiration germanique, l'a emporté sur les besoins du citoyen ${ }^{16}$.

La division entre le Livre II et le Livre III a permis d'utiliser certains concepts de manière certes plus cohérente qu'avant. Ainsi, si le «contrat» est conclu, ce sont les «obligations» qui sont exécutées; ainsi encore, ce n'est pas le contrat auquel il est mis fin, mais la relation contractuelle ou bien les droits et obligations qui résultent du contrat. L'attention nouvelle portée aux droits et obligations dans le Livre III a aussi permis un usage plus rigoureux des termes «créancier» et «débiteur» (de préférence à ceux de «aggrieved party» et «other party» utilisés dans les PDEC). Le fait que le mot «obligation» soit utilisé par référence au droit à l'exécution permet aussi de donner corps à la distinction entre «duty» et «obligations». Enfin, de nombreuses références sont faites aux autres actes juridiques que les contrats («other juridical acts»), parmi lesquels les offres, acceptations, notifications, autorisations, promesses unilatérales. L'inconvénient, c'est que les textes sont souvent assez précis et lourds, avec des distinctions qui n'apparaissent pas évidentes. Ainsi par exemple, la modification du contrat («variation») donne lieu à trois séries de textes, selon qu'elle a lieu soit par un accord, soit par une «notice», soit par le juge en cas de changement de circonstances. Dans les deux premiers cas de figure, les règles s'appliquent à un «droit, obligation ou relation contractuelle» (III-I: 108 ou 109); dans le troisième texte, seule l' «obligation» est visée et le texte précise qu'il peut s'agir d'une «obligation contractuelle ou une obligation provenant d'un acte juridique unilatéral» ((III-I: 110 (2) modification ou la fin du contrat pour changement de circonstances), ce qui exclut les autres sources d'obligations. Tout ceci rend la lecture assez ardue.

\subsection{L'esprit du DCFR}

L'esprit du DCFR n'est pas radicalement différent de celui des PDEC. A certains endroits, il s'en détache pourtant. Cela est notamment illustré par le texte, intitulé "Good faith and fair dealing» (Livre III, art. III-I: 103). Non seulement le texte ne figure pas dans les dispositions générales du Livre I, place hautement symbolique, mais surtout, l'alinéa 3 de cet article semble introduire une restriction nouvelle: la violation du devoir de bonne foi ne donne pas lieu, directement à l'octroi des remèdes (remedies) pour inexécution mais peut empêcher une personne qui viole son obligation d'exercer ou de s'appuyer sur un droit, un remède ou un moyen de défense

16) Lando [5], p. 250. 
qu'elle aurait autrement eu ${ }^{17}$. Dans son article consacré à la structure et aux valeurs du cadre commun de référence, Ole Lando le regrette ${ }^{18}$. A vrai dire, la signification du texte du DCFR est loin d'être évidente. En réalité, elle ne peut être comprise qu'en se référant aux commentaires qui expliquent l'impact de la «policy decision» consistant à employer le mot «duty» et non pas «obligation»» ${ }^{19}$, ce qui restreint l'usage que l'on peut faire du concept de bonne foi. En outre, il faut se référer au mécanisme des obligations implicites (implied term) qui peuvent éventuellement être ajoutées au contrat ${ }^{20}$. Parfois, une «obligation de bonne foi» peut être implicitement contenue dans le contrat; en ce cas, le créancier de cette obligation pourra invoquer l'octroi des remèdes pour inexécution d'une obligation. En revanche, dans les autres cas, le concept de bonne foi ne pourra pas être utilisé par les juges pour rétablir l'équilibre ou la justice contractuels et améliorer le contrat en le rendant plus juste. La bonne foi jouera alors simplement un rôle voisin de celui de l'estoppel/inconsistent behaviour, pour empêcher une personne qui aurait agi en violation du devoir de bonne foi de faire valoir ses droits ${ }^{21}$.

Plus généralement, après avoir dressé une liste des principes directeurs du droit européen, l'introduction du DCFR relève qu'ils entrent nécessairement en conflit entre eux: par exemple, la liberté, plus particulièrement la liberté contractuelle, peut être limitée par les droits fondamentaux (DCFR, introduction, paragraphes 22 et 23). L'introduction explique encore que le DCFR a eu le souci particulier de promouvoir la justice corrective, au sens aristotélicien du terme, et que des clauses générales, telle la bonne foi, permettent précisément d'y parvenir. Pourtant, la bonne foi, parce qu'elle n'est pas admise en tant que telle dans tous les systèmes juridiques, a perdu son rang de principe général. Il est cependant précisé que cela n'interdit pas que les valeurs promues par le principe de bonne foi (par exemple, les pratiques commerciales honnêtes) soient considérées comme fondamentales ${ }^{22}$. La liberté contractuelle est aussi un principe essentiel, avec, pour corollaire, un principe d'«intervention minimum». Le développement de la prospérité économique est également jugé primordial («economic welfare»). Il justifie, en dépit même du principe d'intervention minimum, certaines interventions dans le contrat.

\footnotetext{
17) (3) Breach of the duty does not give rise directly to the remedies for non-performance of an obligation but may preclude the person in breach from exercising or relying on a right, remedy or defence which that person would otherwise have.

${ }^{18)}$ Lando [5], p. 251: "A contract should be regarded as an instrument of co-operation, not as a confrontation of adversaries each seeking his advantage at the expense of the other. It should reflect what Denis Mazeaud calls an esprit collectif (v. les references in note 11, p. 251). It should safeguard solidarity, fairness, reliance and trust, values that everybody, also the weak parties, should adhere to".

${ }^{19)}$ L'Annexe avec les définitions explique la distinction entre «duty» et «obligation» (qui est elle-même une « a duty to perform which one party to a legal relationship, the debtor, owes to another party, the creditor»). Une duty est plus large, et ne s'insère pas forcément dans une relation légale. Il n'y a pas nécessairement de sanction pour une «breach of duty». En résumé, toutes les obligations sont des duties mais toutes les duties ne sont pas des obligations.

${ }^{20)}$ Rappr. PDEC, article 6:102

${ }^{21)}$ Comp. Com., 10 juillet 2007 (cette revue, 2007.1107, notes L. Aynès et D. Mazeaud), rendu au visa de l'article 1134, alinéas $1^{\mathrm{er}}$ et 3 , du code civil: «en statuant ainsi, alors que si la règle selon laquelle les conventions doivent être exécutées de bonne foi permet au juge de sanctionner l'usage déloyal d'une prérogative contractuelle, elle ne l'autorise pas à porter atteinte à la substance même des droits et obligations légalement convenus entre les parties, la cour d'appel a violé, par fausse application, le second des textes susvisés et, par refus d'application, le premier de ces textes».

${ }^{22)}$ von Bar/Clive/Schulte-Nölke [8], Introduction, paragraphe 33.
} 
L'équilibre entre tous ces principes et leurs correctifs est subtil; sans formulation précise, enrichie des commentaires et des notes, la plus grande insécurité règnera.

C'est précisément ce que le groupe AHC/SLC a cherché à faire. L'objectif de notre travail consistait à «éclairer, en les rattachant à leur fondement, l'ensemble des dispositions des Principes du droit européen du contrat, qu'il s'agisse des Principes dans leur état initial ou révisés. Il ne s'agit plus alors de proposer une solution pour chacune des questions du droit du contrat, mais de dégager, en la forme de "principes directeurs» les idées directrices qui, pour autant qu'elles participent d'une culture juridique commune, permettraient d'envisager la construction d'un droit européen

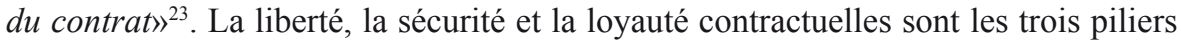
qui se retrouvent dans tous les droits des Etats de l'Union Européenne, en des formes variables.

\section{Le sort de ces travaux}

Le sort des travaux de l'AHC/SLC doit d'abord être envisagé par rapport au projet politique de cadre commun de référence.

Le 18 avril 2008, le Conseil JAI a approuvé un rapport du Comité sur les questions de droit civil relatif à la mise en place d'un cadre commun de référence pour le droit européen des contrats. Le rapport du Comité sur les questions de droit civil définit la position du Conseil sur «quatre aspects fondamentaux du cadre commun de référence»: sa finalité, son contenu, sa portée, ses effets. Sur la base de ce rapport, le Conseil a décidé d'opter pour un «cadre commun de référence complet couvrant le droit général des contrats, y compris le droit des contrats de consommation». Il est précisé que ce CCR n'aurait pas de forme contraignante mais serait «constitué d'un ensemble d'orientations que les législateurs au niveau communautaire utiliseraient, sur une base volontaire, commue une source d'inspiration ou de référence dans le processus législatif ». Il est encore ajouté, faisant ainsi clairement référence aux trois volets que devra contenir le CCR: «Si l'on veut que le cadre de référence commun ait pour effet d'améliorer la qualité et la cohérence de la législation communautaire, il convient que les législateurs communautaires tiennent compte des définitions, principes généraux et règles types qui y figureront lorsqu'ils élaboreront de nouvelles législations ou qu'ils réviseront la législation existante» ${ }^{24}$.

Les principes directeurs pourraient être intégrés au futur CCR, soit en la forme de considérants, soit en celle de textes, intégrés au Livre I. Il n'y avait pas, jusqu'à ce jour, de véritable réflexion sur les principes directeurs du droit des contrats ${ }^{25}$.

\footnotetext{
${ }^{23)}$ Introduction de J.-B. Racine et G. Wicker, dans Association H. Capitant et Société de législation comparée [3].

${ }^{24)}$ Bruxelles, 11 avril 2008, Doc. 8286/08, Justciv 68 Consom 39,

${ }^{25)}$ Certains sont certes implicitement ou même explicitement énoncés; d'autres, nouveaux, sont introduits, tel le principe de non discrimination. Mais le flou existe toujours quant à l'esprit qui anime le DCFR. Les auteurs de l'introduction du DCFR sont du reste eux-mêmes convaincus de l'utilité d'inclure dans le DCFR des principes directeurs, dans une partie séparée (v. introduction précitée, p. 9 et, pour un énoncé des divers principes pris en compte-marché libre, faveur aux consommateurs, aux victimes des discriminations, aux parties faibles. v.p. 13).
} 
Le travail sur la terminologie, pourrait lui aussi être intégré, à côté des de règles modèles (avec une annexe de définition), parmi tous les autres documents destinés à les accompagner, dont les notes et commentaires sous ces règles («essential background information ${ }^{26}$ ).

Enfin, les Principes du droit européen du contrat révisés existent de manière autonome, à côté du DCFR, comme modèle distinct, limité au droit des contrats.

Pour finir, on insistera sur le fait que les travaux réalisés par le groupe AHC/SLC ont une valeur propre, indépendante du projet politique de cadre commun de référence. Ils constituent un nouvel outil pour tous ceux qui s'intéressent à l'évolution du droit des contrats en Europe ainsi que pour tous les comparatistes, qu'ils soient universitaires ou praticiens, français ou étrangers. Ils ont été élaborés par des équipes, certes majoritairement composées de juristes français, mais qui ont eu le souci constant de ne favoriser aucune tradition juridique, aucun droit national.

\section{Références}

1. Association H. Capitant et Société de législation comparée: European Contract Law - Materials for a Common Frame of Reference: Terminology, Guiding Principles, Model Rules, Sellier (2008)

2. Association H. Capitant et Société de législation comparée: Projet de cadre commun de référence. Terminologie contractuelle commune, coll. Droit privé comparé et européen 6 (2008)

3. Association H. Capitant et Société de législation comparée: Projet de cadre commun de référence. Principes contractuels communs, coll. Droit privé comparé et européen 7 (2008)

4. Groupe d'étude sur la justice sociale en droit privé européen: Manifeste pour une justice sociale en droit européen des contrats, R. T.D.Civ. No. 4, 713-734 (2005)

5. Lando, O.: The Structure and the Legal Values of the Common frame of Reference (CFR). ERCL 3, 245-256 (2007)

6. Société de législation comparée: Principes du droit européen du contrat, coll. Droit privé comparé et européen 2 (2003)

7. Study Group on Social Justice in European Private Law: Social justice in European contract law: a Manifesto. European Law journal 10(6), 653-674 (2004)

8. von Bar, C., Clive, E., Schulte-Nölke, H.: Principles, Definitions and Model Rules of European Private Law. Draft Common Frame of Reference, Sellier (2008)

26) V. supra; adde von Bar/Clive/Schulte-Nölke [8], paragraphe 72, p. 35. 